\title{
A Microarchitecture Design Methodology to Achieve Extreme Isotropic Elastic Properties of Composites Based on Crystal Symmetries
}

\author{
Nestor Rossi ${ }^{\mathrm{a}}$, Juan M. Podestáb ${ }^{\mathrm{b}}$,Facundo Bre ${ }^{\mathrm{a}}$, Carlos G. Méndez ${ }^{\mathrm{a}}$, Alfredo E. Huespe ${ }^{\mathrm{a}, \mathrm{c}, *}$ \\ ${ }^{a}$ Centro de Investigación de Métodos Computacionales (CIMEC), UNL, CONICET. Predio "Dr. Alberto \\ Cassano", Colectora Ruta Nacional 168 s/n, Santa Fe, 3000, Argentina \\ ${ }^{b}$ Computational Mechanics Laboratory (LAMEC-IMIT). Northeast National University (UNNE) - National \\ Scientific and Technical Research Council (CONICET). Resistencia, 3500, Argentina \\ ${ }^{c}$ Universitat Politècnica de Catalunya (UPC) - BarcelonaTech. Barcelona, 08034, Spain
}

\begin{abstract}
The present contribution describes an optimization-based design technique of elastic isotropic periodic microarchitectures with crystal symmetries aiming at the realization of composites with extreme properties. To achieve this goal, three consecutive procedures are followed: i) a series of inverse homogenization problems with symmetry constraints, ii) a correlation analysis between symmetries and effective elastic properties of the attained microarchitectures, and, iii) the pattern resemblance recognition of these topologies and their redesign, by adopting microstructures with two length-scales, through optimized parametric geometries. This paper is devoted to assessing the third procedure because the first two procedures have been evaluated in previous works of the authors, and here they are only summarized.

By applying the methodology, two plane group symmetries are assessed to define two families of 2D periodic parameterized microarchitecture. Once the parameters have been optimized, the resulting composites achieve elastic isotropic properties close to the whole range of the theoretically estimated bounds. Particularly, an unprecedented microstructure attaining the theoretical maximum stiffness is reported. Starting from these parameterized topologies, simple, one-length scale and easily manufacturable geometries are defined. One of the so-designed microarchitectures has been manufactured and tested, displaying an effective Poisson's ratio of -0.90 simultaneously with a high shear modulus.

Keywords: extreme elastic properties, parameterized microstructure designs, topology optimization, multi-objective optimization, crystallographic symmetries, auxetic composite
\end{abstract}

\footnotetext{
* Corresponding author

Email address: ahuespe@cimec.unl.edu.ar (Alfredo E. Huespe)
} 


\section{Introduction}

${ }_{31}$ The synthesis of composites with unusual effective properties constitutes an important goal in man-

$$
G^{u}(K)=\frac{\omega^{u}\left(f_{1} G_{1}+f_{2} G_{2}\right)+G_{1} G_{2}}{G_{2} f_{1}+G_{1} f_{2}+\omega^{u}} \quad ; \quad \omega^{u}=-G_{1}+\frac{\alpha^{u} y_{K}}{\beta^{u} y_{K}-\gamma^{u}} ;
$$

and below by the lower bound $G^{l}(K)$ defined by:

$$
G^{l}(K)=\frac{\omega^{l}\left(f_{1} G_{1}+f_{2} G_{2}\right)+G_{1} G_{2}}{f_{1} G_{2}+f_{2} G_{1}+\omega^{l}} \quad ; \quad \omega^{l}=-G_{1}+\frac{\alpha^{l}\left(\frac{1}{y_{K}}+\frac{1}{K_{1}}\right)}{\beta^{l}\left(\frac{1}{y_{K}}+\frac{1}{K_{1}}\right)-\gamma^{l}} ;
$$

where

$$
y_{K}=-K_{1} K_{2} \frac{K-\frac{1}{\left(\frac{f_{1}}{K_{1}}+\frac{f_{2}}{K_{2}}\right)}}{K-\left(f_{1} K_{1}+f_{2} K_{2}\right)}\left(\frac{f_{1}}{K_{1}}+\frac{f_{2}}{K_{2}}\right) ;
$$

47 and the coefficients $\alpha, \beta, \gamma$ depend on $K_{1}, G_{1}, K_{2}$, and $G_{2}$.

48 It is usually assumed that material moduli of the phases are related by a stiffness ratio or contrast 49 factor $r=K_{1} / K_{2}=G_{1} / G_{2}$. Particularly, for a composite whose phases are characterized by the 50 elastic parameters: $K_{1}=5 / 7, G_{1}=5 / 13, f_{1}=0.5$, and $r=200$; the coefficients $\alpha, \beta, \gamma$ result: ${ }_{51} \quad \alpha^{l}=0.4107, \beta^{l}=1.0653, \gamma^{l}=0.0110, \alpha^{u}=-0.1256, \beta^{u}=-0.2200$ and $\gamma^{u}=2.0 \times 10^{-4}$. The ${ }_{52}$ upper and lower bounds for the effective bulk modulus coincide with the Hashin-Shtrikman bounds $53 \quad[4]$

$$
K_{H S}^{u}=K_{1}+\frac{f_{2}}{\frac{1}{K_{2}-K_{1}}+\frac{f_{1}}{K_{1}+G_{1}}} \quad ;
$$




$$
K_{H S}^{l}=K_{2}+\frac{f_{1}}{\frac{1}{K_{1}-K_{2}}+\frac{f_{2}}{K_{2}+G_{2}}} .
$$

The CG-bounds are plotted in Figure 1. Alternatively to the $K-G$ space of effective properties, CG-bounds can be plotted in the $E-\nu$ space, with $E$ being the effective Young modulus and $\nu$ the Poisson's ratio, as it is also depicted in Figure 1. In this work, it is indistinctly made reference to both spaces according to what is more convenient to perform the desired analysis. Moreover, composites with effective properties lying on the analytical CG-bounds are herein called extreme materials.

Figure 1 also indicates the regions of these bounds defining composites with extreme properties displaying interesting features that could be technically exploited. For example, the composites whose properties stay on the CG-bounds along the gray zone are auxetics (i.e., negative Poisson's ratios: $\nu<0$ ). Also, the composite with the most negative Poisson's ratio, represented by point B, corresponds to the point on the CG-bounds which is tangent to the straight lines crossing the origin of coordinates, in the $K-G$ space. The stiffest isotropic composite which could be attained with phases $M_{1}$ and $M_{2}$, and a given volume fraction $f_{1}$ corresponds to the upper-right vertex. Another specific class of composites is the isotropic bimode materials, which, according to [5], count with a high $K / G$ ratio. They are located on and near the lower bound, with minimum $\mathrm{G}^{1}$. Bimode materials have been proposed for acoustic cloaking applications in [6-9]. Some of these remarkable features (auxetic composites region and minimum Poisson's ratio) are more clearly enlightened in the $E-\nu$ space.

For a long time, it has been of interest to know if extreme materials are realizable. Recent contributions aiming at this objective for non-periodic microstructures are $[10,11]$ and references cited therein. The assemblage of coated spheres [12, 13], Vigdergauz structures [14], and the composite class reported by Sigmund [15] are materials attaining the theoretical maximum $K$. Additionally, maximum and minimum stiffness materials (upper-right and lower-left corners of the CG-bounds in the $K-G$ space) can be achieved by sequential laminates $[16,17]$, specifically by rank-3 laminates [18]. Besides, the closest known solution to the Walpole point (lower-right corner in $K-G$ space), is the hexagon structure reported by Sigmund [15]. A more detailed discussion on the effect that different microarchitecture topologies have to attain extreme materials can be found in [19]. Despite the previously mentioned families of microstructures, it has not yet been reported that the entire CG-bounds are attainable. Furthermore, excluding Vigdergauz structures, these

\footnotetext{
${ }^{1}$ It is worth remarking that void is needed as the soft phase to achieve null eigenvalues of the constitutive tensor, achieving strict bimode materials.
} 


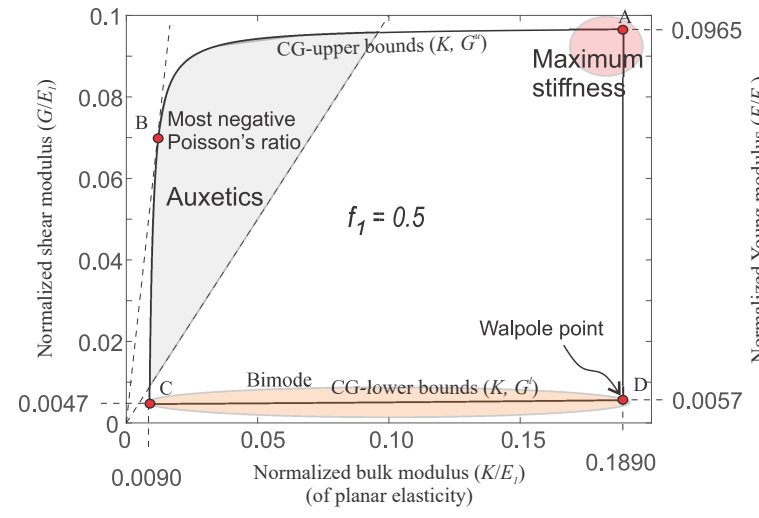

(a)

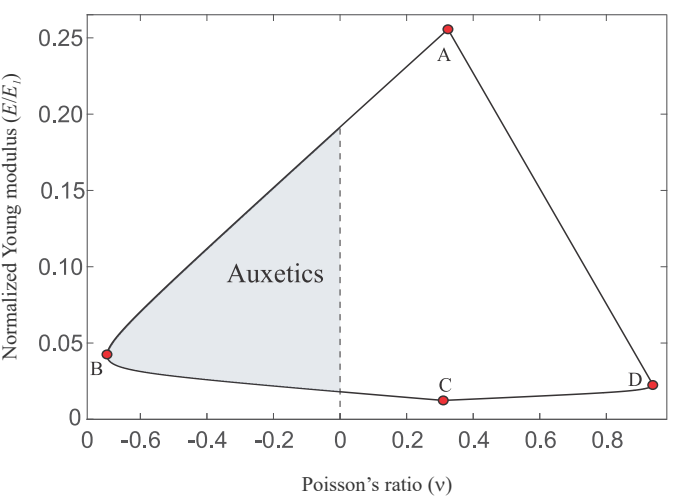

(b)

Figure 1: Analytic bounds of effective properties for two-phase isotropic composites according to [3] (CG-bounds). Plane elasticity. a) Bounds in the space $(K, G)$; b) same bounds in the space $(E, \nu)$. Properties of phases $M_{1}$ and $M_{2}$ are $K_{1}=5 / 7 ; K_{2}=K_{1} / r ; G_{1}=5 / 13 ; G_{2}=G_{1} / r, r=200\left(E_{1}=1, E_{2}=E_{1} / r, \nu_{1}=\nu_{2}=0.3\right)$. Volume fraction of phase $M_{1}$ is $f_{1}=0.5$.

microarchitectures achieving the theoretical CG-bounds correspond to topologies displaying more than one length-scale, which seems to benefit the possibility of attaining an extreme response but seriously limits the manufacturability and use of the composites in actual applications. Additionally, as recently reported by Milton [20], auxetic biphasic composites, with void as the soft phase and effective Poisson's ratio tending to -1 , besides of having a vanishing bulk modulus, generally present a vanishing shear modulus.

The microarchitecture design methodology reported in this work pursues the attainment of simple two-length-scale parameterized periodic topologies, defined by only a few design parameters. These microstructures should be able to attain properties near to the whole theoretical bounds and should satisfy crystal symmetries. The adequate symmetries to be imposed on these topologies are assessed as an additional result from the same methodology. Additionally, it is shown that the designed auxetic microstructures retain high shear stiffness in the limiting case of the stiffness ratio between phases going to infinite.

To achieve this target, an optimization-based design methodology consisting of three consecutive procedures is proposed and is briefly described in Section 2. The remaining part of the paper is organized as follows. Section 3 describes a brief overview of inverse homogenization problems assuming crystal symmetries. In Section 4, the geometrical patters regarding material distribution are analyzed and the novel parameterized microstructures are introduced. Section 5 presents a multi-objective optimization study to validate the extreme properties attainable by the parameterizations. Results are presented and discussed in Section 6. Section 7 explores the influence that 
similar one-length-scale topologies have on the microarchitecture capability to achieve extreme effective properties. Additionally, in the same Section, the experimental results of a manufactured specimen is reported. Finally, Section 8 establishes the main conclusions of this work.

\section{Microstructure design methodology}

The main idea behind the present approach is to enforce predefined crystallographic symmetries throughout the optimal topology design process of composite microarchitectures to attain unusual effective elastic properties. Recently, several works have reported the use of symmetries in microstructure design [21-29]. The periodic pattern assumed for the microarchitecture topology jointly with the prescribed symmetries generates microarchitectures that can be assimilated to crystals.

Inspired by this concept, the proposed design methodology is based on three successive procedures summarized in the following items:

i) the first step in the design procedure consists of solving several topology optimization problems with an appropriate mathematical formulation posed as an inverse homogenization problem [30]. The target properties of these optimization problems are defined according to the CG-bounds; a point on the CG-bounds defines the effective target properties of the optimization problem which is solved. The attained microarchitecture configurations are forced to have different crystal symmetries that have to be consistent with the symmetry of the target effective properties. This procedure is repeated for several points on the CG-bounds.

ii) The elastic properties of the designed microarchitectures are evaluated by correlating the attained closeness to the CG-bounds with the imposed symmetries, see [31-34]. Conclusions on the most suitable crystal symmetries to approach the full range of the CG-bounds are assessed.

iii) The patterns of the topologies obtained with those crystal symmetries are identified and redefined through a geometrical parameterization, by adopting a two-scale material distribution, respecting the symmetries ${ }^{2}$ that inspired them. Finally, with the proposed configurations, a multi-objective optimization problem is solved to attain the set of parameters defining

\footnotetext{
${ }^{2}$ It is commonly observed that solutions of the inverse homogenization problem, in step 1 of item i), may provide microarchitectures with higher symmetry to the imposed one. In these cases, the procedure in item iii) adopts this higher symmetry.
} 
the microarchitectures whose effective properties are the closest ones to those of extreme materials.

The above mentioned procedures in items $i$ ) and ii) have been studied and reported by the authors in previous works [31-34] and are summarized in Section 3 for the sake of completeness, while the topic mentioned in item iii) is developed in detail in Sections 4 and 5 and constitutes the main contribution of this paper.

\section{Inverse homogenization problems assuming crystal symmetries}

A basic micro-cell $\Omega_{\mu}$ of a two-phase periodic composite is considered, such as is schematically shown in Figure 2. The phases $M_{1}$ and $M_{2}$ occupy the domains $\Omega_{\mu}^{1}$ and $\Omega_{\mu}^{2}$, respectively. A characteristic function $\chi(\boldsymbol{y})$, in $\Omega_{\mu}$, identifies the positions where the phase $M_{1}$ is placed, such as indicated in the same Figure.

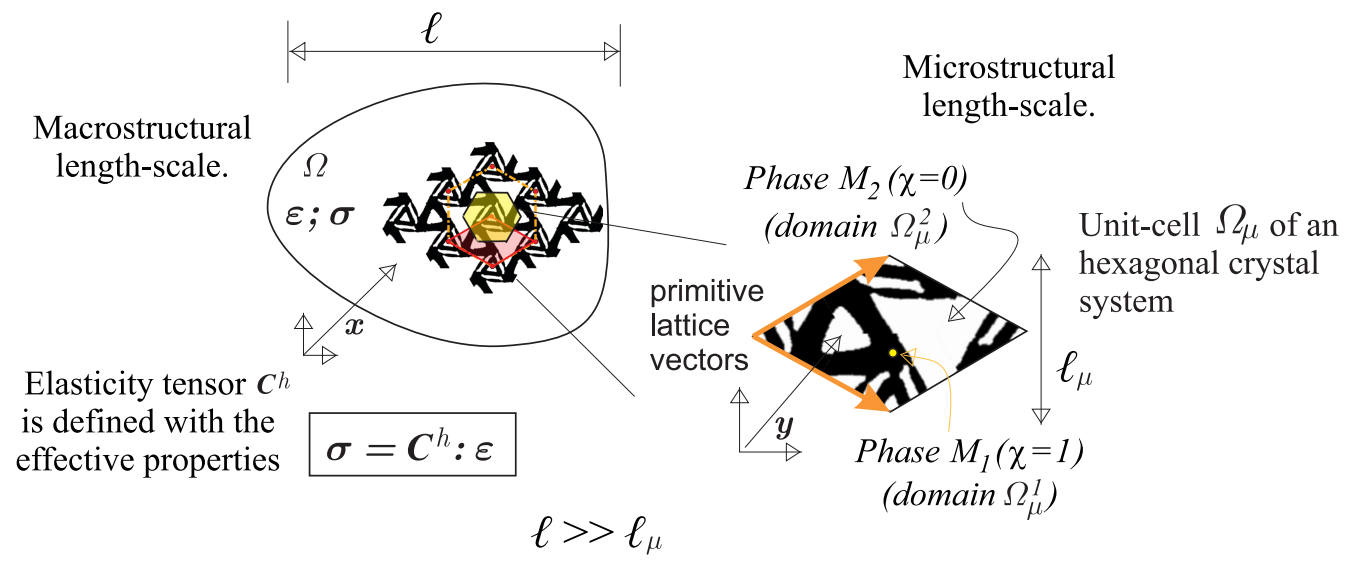

Figure 2: Inverse homogenization problem to determine the characteristic function $\chi$ of a periodic composite whose microstructure has hexagonal crystal symmetry. The domain $\Omega_{\mu}$ adopted for the topology design problem is a unit cell of the underlying hexagonal Bravais lattice. The periodicity directions are defined by the primitive vectors of the lattice. The effective elasticity tensor $\boldsymbol{C}^{h}$ relating the macrostrain $\boldsymbol{\varepsilon}$ with the macrostress $\boldsymbol{\sigma}$ is evaluated with a computational homogenization technique using the same cell.

Following the procedure established in [31, 33], a micro-architecture inverse design problem is formulated as a topology optimization problem [35-37] to find a composite whose effective properties are a given target pair of values $K^{t}$ and $G^{t}$. A sequence of target values $K^{t}$ and $G^{t}$ on the CG-bounds are chosen, and the corresponding topology optimization problems are solved to find the microarchitectures providing the closer elastic properties to the CG-bounds. In [31-34], the single length-scale microarchitectures are found using a topology optimization algorithm based 
Table 1: Hexagonal (2D) Crystal System. Compatible point and plane groups. The elasticity matrix is isotropic and is represented in column 1 , with the coefficient $C_{11}$ and $C_{12}$ characterizing the elastic properties. The conventional Kelvin notation for fourth order tensors of plane elasticity is used.

\begin{tabular}{|c|c|c|c|c|c|c|c|}
\hline & \multicolumn{2}{|c|}{ Elasticity } & $\begin{array}{l}\text { Crystal } \\
\text { system }\end{array}$ & $\begin{array}{l}\text { Point } \\
\text { group }\end{array}$ & $\begin{array}{l}\text { Plane } \\
\text { group }\end{array}$ & $\begin{array}{c}\text { Compatible } \\
\text { Bravais lattice }\end{array}$ & $\begin{array}{l}\text { Wigner-Seitz and } \\
\text { primitive unit cell }\end{array}$ \\
\hline \multirow{5}{*}{$\begin{array}{c}C_{11} \\
C_{12} \\
0\end{array}$} & \multirow{5}{*}{$\begin{array}{c}C_{12} \\
C_{11} \\
0\end{array}$} & \multirow{5}{*}{$\left.\begin{array}{c}0 \\
0 \\
C_{11}-C_{12}\end{array}\right]$} & \multirow{5}{*}{ hexagonal } & 3 & p3 & \multirow{5}{*}{ hexagonal } & \multirow{5}{*}{$\bullet$} \\
\hline & & & & \multirow{2}{*}{$3 \mathrm{~m}$} & $p 3 m 1$ & & \\
\hline & & & & & p31m & & \\
\hline & & & & 6 & $p 6$ & & \\
\hline & & & & $6 \mathrm{~mm}$ & p6mm & & \\
\hline
\end{tabular}




\subsection{Computational homogenization}

The effective properties of the composites are evaluated in the primitive cell of the so-designed periodic microstructure, which is coincident with the same domain taken for the topological optimization problem. This procedure defines the tensor $\boldsymbol{C}^{h}$ and is schematically shown in Figure 2.

The computational homogenization follows a conventional technique that is well-described in the literature, see for example [43] and references cited therein. Periodic boundary conditions on the displacement fluctuation field, along the primitive vectors of the underlying Bravais lattice, are imposed.

\subsection{Solutions attained with the inverse homogenization problems}

Results of topology optimization problems using the above-mentioned methodology to attain near extreme effective isotropic properties, which show different plane groups compatible with the hexagonal crystal system, have been reported in [31,33]. These solutions are obtained by restricting the topology to have only one length-scale.

The analysis in these works leads to the following conclusions. Plane groups $p 3, p 31 m$ and $p 6$ are well-suited for the top-left region in the $K-G$ space, while plane groups $p 3 m 1$ and $p 6 m m$ are more convenient for the bottom-right sector, such as shown in Figure 3. The same Figure shows some of the optimum microstructures, denoted $\mathrm{T} 1, \ldots, \mathrm{T} 7$, whose effective elastic properties approximate the bounds, see Figures 3a and b.

The microarchitectures in Figure 3 display common patterns motivating the present work, as it is explained in the next Section.

A further discussion about the sensitivity of each plane group consistent with the hexagonal crystal system to attain extreme properties in different sectors of the CG-bounds appears in [33]. The reader is addressed to this reference work for additional details about this specific point related to the microarchitecture design problem.

\section{Geometrical pattern recognition of microstructures and their parameterization}

In small sectors of the CG-bounds, a large number of solutions are obtained with the inverse homogenization problems. Based on these results, and noting that the enforcement of the symmetry constraints promotes the attainment of solutions displaying similar topologies for small changes of the targets, it is possible to recognize a geometrical material distribution that represents the composite microstructure for that given sector of the CG-bounds. 


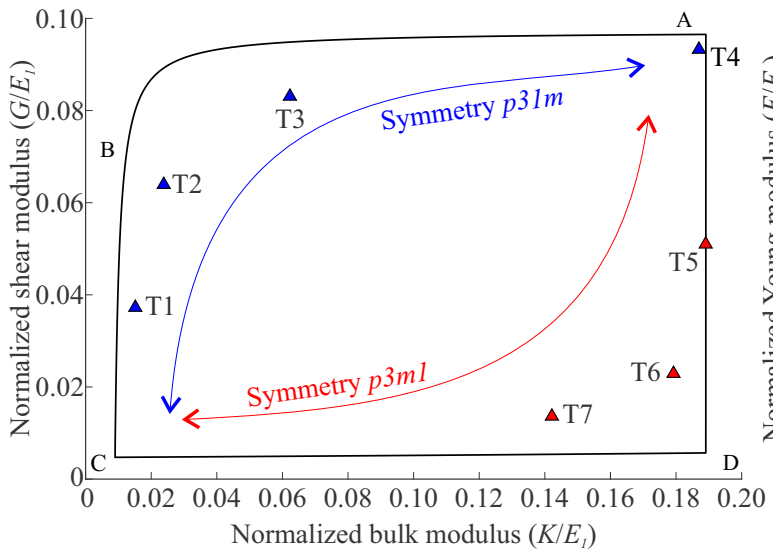

(a)

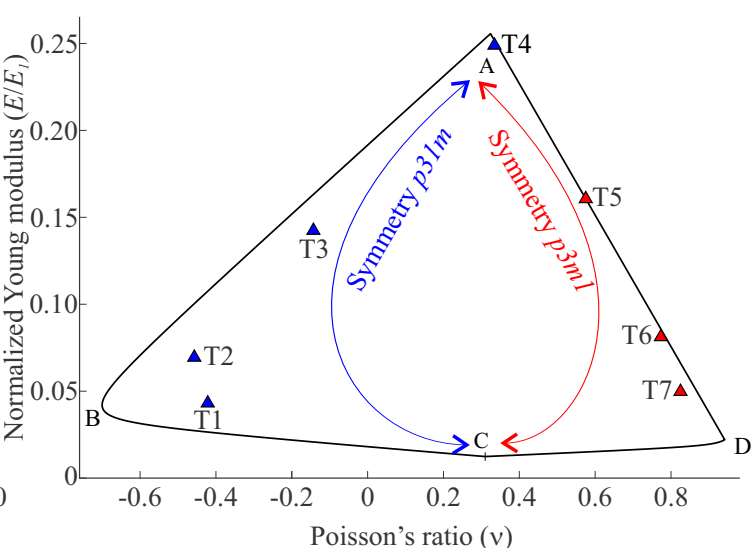

(b)

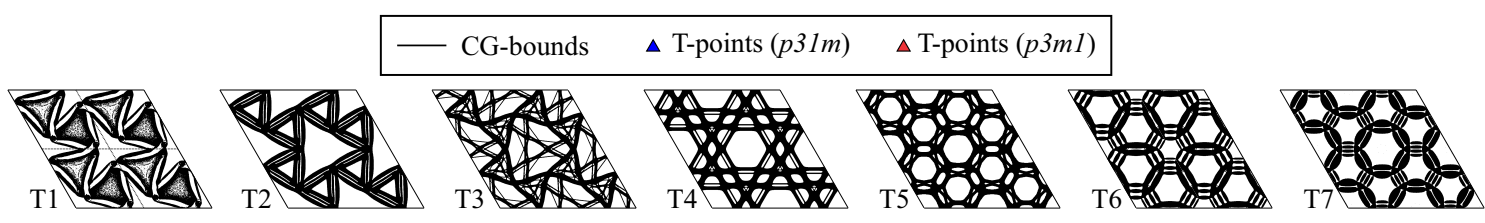

(c)

Figure 3: CG-bounds of well-ordered biphasic composites with isotropic properties in the space of effective normalized properties: a) $K-G$, and b) $E-\nu$. Composites with stiff phase volume fraction: $f_{1}=0.5$. Elastic properties of the stiff phase: $E_{1}=1, \nu_{1}=0.3$. Stiffness ratio between phases: $r=2 \times 10^{2}$. c) Microstructures obtained by topology optimization, a procedure formulated as a mathematical problem (inverse homogenization problem) $(2 \times 2$ unit cells), instances $\mathrm{T} 1$ to $\mathrm{T} 7$ reported in [33].

By employing this approach, it is possible to assess common geometrical patterns that suggest a road to reduce the original complex morphologies, provided by the topology optimization algorithm, to simpler ones. Specifically, simpler representative microarchitectures are adopted, based on the geometrical parameterization of the material configurations.

The assessment of some common geometrical patterns in the microstructures displayed in Figure $3 \mathrm{c}$ can be summarized as follows:

- Figure 4 replicates the microarchitectures $\mathrm{T} 1, \ldots, \mathrm{T} 4$ with their corresponding unit cells. It can be observed that these microstructures have a material distribution notoriously resembling a pair of triangles with symmetry $p 31 m$, rotating relatively one to other as the target elastic properties move from the lower-left to the upper-right CG-bounds (see Figure 3a). These triangles are highlighted in dashed red lines in the unit cells.

- The microarchitectures T5,.., T7 in Figure 4 have a material distribution resembling a pair of irregular hexagons, highlighted in dashed red lines, with symmetry $p 3 m 1$, whose shape 


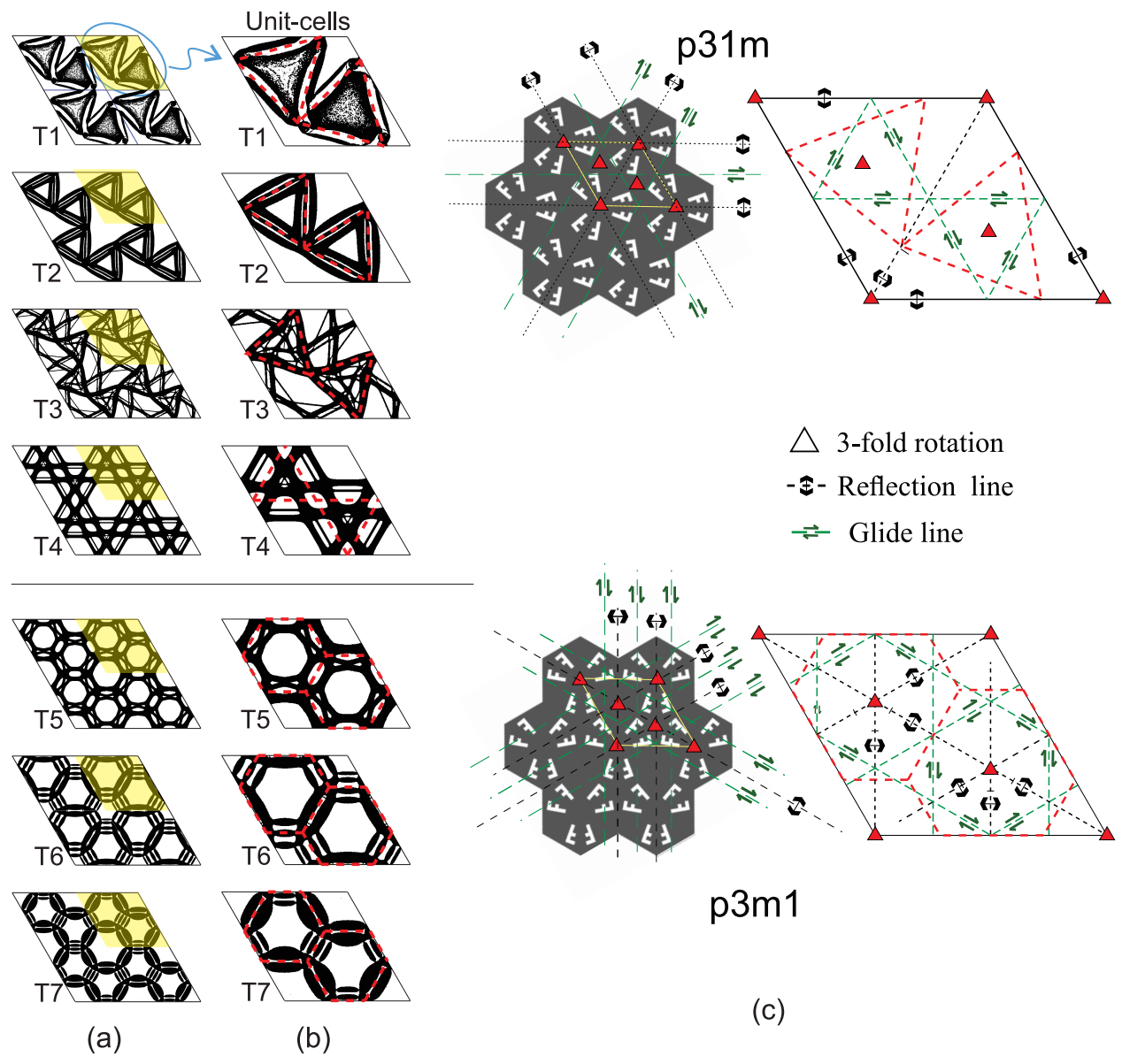

Figure 4: Geometrical pattern recognition. a) Microarchitecture configurations T1 to T7. b) unit-cells ; c) symmetry elements of plane groups $p 3 m 1$ and $p 31 \mathrm{~m}$. Dashed red lines indicate the underlying material distribution pattern.

continuously changes as the target elastic properties move from the upper-right to the lowerleft CG-bounds.

- Additionally, a bar-like substructure recurrently appears in the sides of these polygons, a feature already noted by Sigmund [15] when trying to attain the Walpole point.

- The symmetry elements of both plane groups $p 31 m$ and $p 3 m 1$ are shown in Figure 4 . Note that they have analogous symmetry elements: a three-fold rotational symmetry and three mirror planes. However, the mirror planes of the $p 3 m 1$ plane group intersect the WeignerSeitz cell differently to that of the $p 31 \mathrm{~m}$ plane group. This variant makes a big difference to obtain suitable results in different regions of the CG-bounds.

These observations motivate the development of two families of parameterized microstructures, 
with symmetries $p 31 m$ and $p 3 m 1$. As explained later, the symmetry of each family defines the region of the CG-bounds where the parameterized family is appropriate to approach the extreme properties.

The parameterized microstructures are defined by three constituents, see Figure 5 . Further to the soft and stiff phases, and inspired by the microstructures adopted in Sigmund's work [15], the bars in the sides of these polygons are replaced by laminated components. Each laminate is assumed as a sequence of layers of the same soft and stiff phases of the original biphasic composite. The width of each layer is much smaller than the unit cell size, introducing in the geometrical configuration a lower length scale respect to that of the cell size. A similar procedure has been reported recently by [44].

The geometrical parameters defining the material configurations of the two proposed microstructure families are described in Figure 5. Black regions identify the stiff phase, white regions are the soft phase, and gray regions represent the laminated components. The laminate grayscale identifies the volume fractions of the laminate and the dashed red lines indicate the layer directions of the laminate.

In the configuration $p 31 m$, an angle $\alpha$ characterizes the rotation of two identical equilateral triangles. In the configuration $p 3 m 1$, the dimensionless parameter $d$ defines two identical irregular hexagons.

The configuration $p 31 m$ is defined by three parameters, $\alpha, \gamma$ and $t$, and the configuration $p 3 m 1$ is defined by five parameters, $d, \gamma_{1}, \gamma_{2}, \gamma_{3}$, and $t$. In both cases, the thickness $t$ of the laminate areas, common for all of them, comes as a result of the values of the other parameters, once the target volume fraction $f_{1}^{t}$ is imposed.

\subsection{Computational homogenization of the parameterized microarchitectures}

A summary of the numerical implementation technique for assessing the effective properties of the parameterized microarchitectures is given next. These properties are assessed through computational homogenization, such as explained in Subsection 3.2. However, in this case, to increase the numerical accuracy, all the computed unit-cells are modeled employing conformal meshes of finite elements that exactly capture the geometry of the interfaces. Thus, a procedure has been developed to adjust the finite element mesh to every particular tested combination of parameters. At the length-scale corresponding the unit-cell observation, the laminate component is modeled as a homogeneous orthotropic elastic phase with an effective elasticity tensor with components: 
(a) $\mathrm{p} 31 \mathrm{~m}$

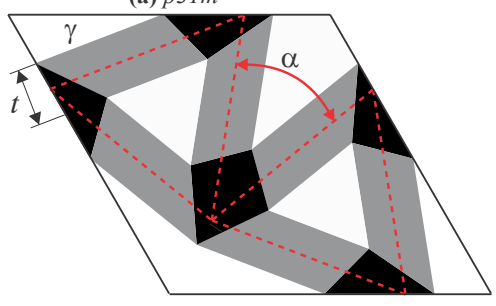

(b) $p 3 m 1$

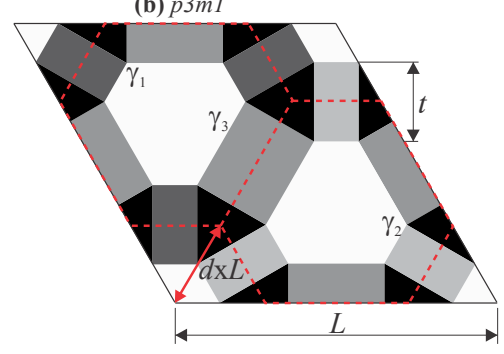

tiff phase

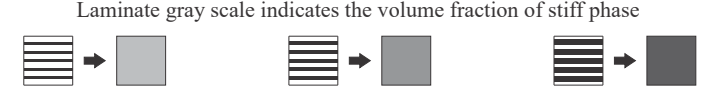

Figure 5: Parameterizations of the unit-cell geometries. Configurations: a) $p 31 m$ and b) $p 3 m 1$. Black and white domains represent stiff and soft phases. Gray domains represent laminates constituted of a sequence of stiff and soft layers parallel to the red lines. The volume fractions of the laminate phases are determined by the parameters $\gamma, \gamma_{1}, \gamma_{2}$, and $\gamma_{3}$, respectively.

$$
\begin{aligned}
& C_{11}=\frac{\left(K_{1}+G_{1}\right)\left(K_{2}+G_{2}\right)}{(1-\gamma)\left(K_{1}+G_{1}\right)+\gamma\left(K_{2}+G_{2}\right)} ; \\
& C_{12}=\frac{\left(K_{1}-G_{1}\right)\left(K_{2}+G_{2}\right)+2(1-\gamma)\left(K_{2} G_{1}-K_{1} G_{2}\right)}{(1-\gamma)\left(K_{1}+G_{1}\right)+\gamma\left(K_{2}+G_{2}\right)} ; \\
& C_{22}=C_{11}+\frac{4 \gamma(1-\gamma)\left(K_{1}-K_{2}\right)\left(G_{1}-G_{2}\right)}{(1-\gamma)\left(K_{1}+G_{1}\right)+\gamma\left(K_{2}+G_{2}\right)} ; \\
& C_{33}=\frac{2 G_{1} G_{2}}{(1-\gamma) G_{1}+\gamma G_{2}} ;
\end{aligned}
$$

where the vertical direction in this case (axis 2) is parallell to the laminate direction.

\subsection{Range of parameters, topological changes and special cases}

The range of the parameters defining the microarchitecture geometries are limited by evident geometrical reasons and depends on the plane group characterizing the topology.

Regarding the $p 31 m$ family of microstructures, the criteria to define the parameters bounds is the topological change that occur if some of the soft material regions collapse into a point. For instance, in the microarchitecture displayed in Figure 5a, if the parameter $\alpha$ reaches a sufficiently small value, the soft material in the vertices of the the cell disappear. Similar effects happen if the stiff material volume fraction $f_{1}$ increases or the laminate volume fraction $\gamma$ decreases, which 
also affect the soft material triangles in the interior of the unit cell. Despite this, there are obvious values that cannot be surpassed: $0^{\circ} \leq \alpha \leq 120^{\circ}, 0 \leq \gamma \leq 1$, and $0 \leq f_{1} \leq 1$.

In the case of the $p 3 m 1$ family of microstructures analogous criteria is used, although laminated regions are allowed to disappear. By taking the microarchitecture displayed in Figure 5b, the $d$ parameter reaches the lower bound when laminates regions with volume fractions $\gamma_{1}$ and $\gamma_{2}$ vanish. In this case, if $\gamma_{3}=0.5$, the Sigmund's triangle structure reported in [15] is obtained. On the contrary, if $d$ increases, the laminate region of $\gamma_{3}$ may vanishes. However, this is not a criterion to limit the upper value of $d$, which may increase up to $d=0.5$, since in this situation, laminates with $\gamma_{1}$ and $\gamma_{2}$ get aligned $^{3}$. Two characteristics of this parameterization are noted: i) $p 6 m m$ symmetry is attained if $\gamma_{1}=\gamma_{2}$, and ii) for $\gamma_{1}=\gamma_{2}=\gamma_{3}=0.5$ and $d=1 / 3$, the above mentioned Sigmund's hexagon structure is recovered. The limits that cannot be surpassed in this parameterization are: $0 \leq d \leq$ $0.5,0 \leq \gamma_{1} \leq 1,0 \leq \gamma_{2} \leq 1,0 \leq \gamma_{3} \leq 1$, and $0 \leq f_{1} \leq 1$. In addition, note that both configurations coincide if, having every $\gamma$ the same value, the parameter $d=0.5$ and $\alpha=120^{\circ}$.

\section{Optimized parametric microstructures}

The optimal set of parameters defining the composites whose effective properties are closer to the CG-bounds, are assessed through a multi-objective optimization technique. The space of design variables $\boldsymbol{p}$, where $\boldsymbol{p}=\{\alpha, \gamma, t\}$ for the $p 31 \mathrm{~m}$ parameterization and $\boldsymbol{p}=\left\{d, \gamma_{1}, \gamma_{2}, \gamma_{3}, t\right\}$ for the p3m1 parameterization, is the admissible set of geometrical parameters $\mathcal{V}$ identifying the microstructure configuration, which is bounded by the criteria specified in the previous subSection 4.2 .

According to Figure 6, the full CG-bounds could be approximated by solving four bi-objective optimization problems formulated as follows:

$$
\begin{aligned}
\min _{\boldsymbol{p} \in \mathcal{V}} & {\left[h_{1}(\boldsymbol{p}), h_{2}(\boldsymbol{p})\right] } \\
\text { subject to: } & f_{1}(\boldsymbol{p})-f_{1}^{t}=0,
\end{aligned}
$$

where $h_{1}(\boldsymbol{p})$ and $h_{2}(\boldsymbol{p})$ are the objective functions, while $f_{1}(\boldsymbol{p})$ and $f_{1}^{t}$ are the cell volume fraction and the target volume fraction respectively. The objective functions, $h_{1}, h_{2}$, for each problem are specified as follows:

- Problem 1: to attain the boundary A-B, $h 1=\nu$ and $h 2=-E$;

\footnotetext{
${ }^{3}$ Note that orthogonality of the laminate axis and the stiff material on the interphase is lost when $d$ is increased once laminate with $\gamma_{3}$ vanishes.
} 
- Problem 2: to attain the boundary B-C, $h 1=\nu$ and $h 2=E$;

- Problem 3: to attain the boundary C-D, $h 1=-\nu$ and $h 2=E$;

- Problem 4: to attain the boundary A-D, $h 1=-\nu$ and $h 2=-E$;

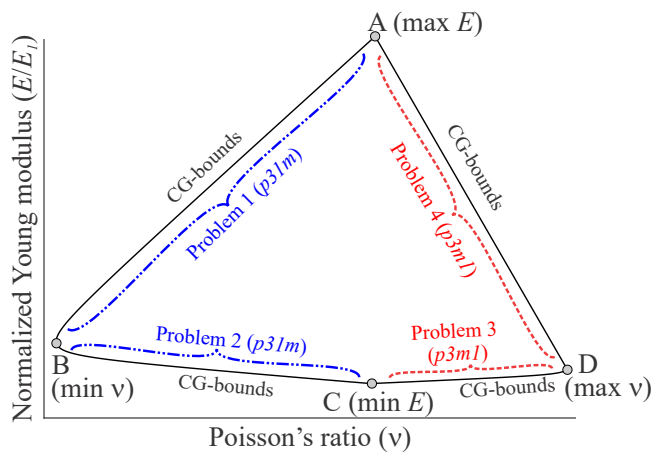

Figure 6: Four bi-objective optimization problems formulated to attain the CG-bounds.

Configurations $p 31 m$ are used to solve problems 1 and 2 , then

$$
f_{1}=\frac{\gamma \Omega_{l a m}(\alpha, t)+\Omega_{1}(\alpha, t)}{\Omega_{\mu}} .
$$

Instead configurations $p 3 m 1$ are used to solve problems 3 and 4 , where

$$
f_{1}=\frac{\gamma_{1} \Omega_{\text {lam } 1}(d, t)+\gamma_{2} \Omega_{\text {lam } 2}(d, t)+\gamma_{3} \Omega_{\text {lam } 3}(d, t)+\Omega_{1}(d, t)}{\Omega_{\mu}} .
$$

In both cases, $\Omega_{\text {lam }}, \Omega_{\text {lam } 1}, \Omega_{\text {lam } 2}$, and $\Omega_{\text {lam } 3}$ are the total areas occupied by the laminate materials and $\Omega_{\mu}$ is the area of the unit cell.Full expressions of the volume fraction constraints (11) and (12) are described in the Appendix.

Solutions of problems 1 to 4 are given through the set of non-dominated optimal design variables, known as Pareto fronts. Given the contradictory nature of these multi-objective optimization problems, the set of parameters defining each Pareto front is found using the NSGA-II (nondominated sorting genetic algorithm-II) [45].

\section{Results and discussion}

The Pareto fronts resulting from problems 1 to 4 using the parametrized microstructures previously defined are plotted in Figures 7a and b. For each of these problems, an average of approximately 275 non-dominated optimal solutions have been obtained. Thus, around 1100 points of attainable 


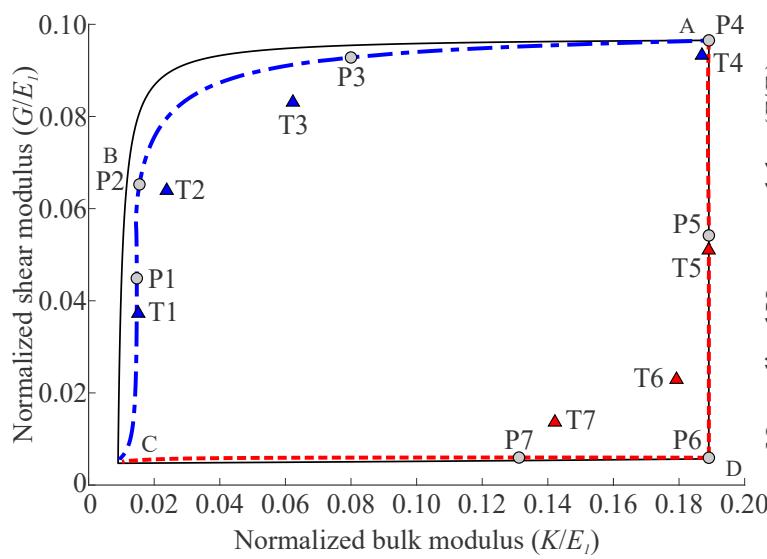

(a)

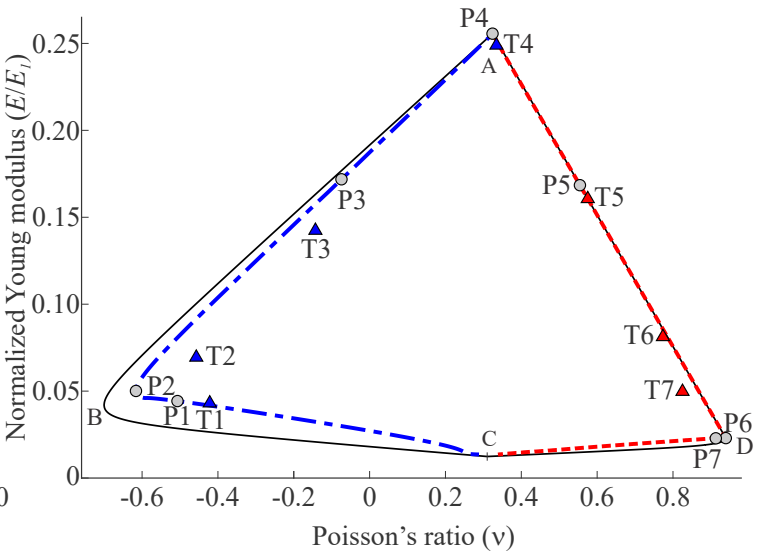

(b)

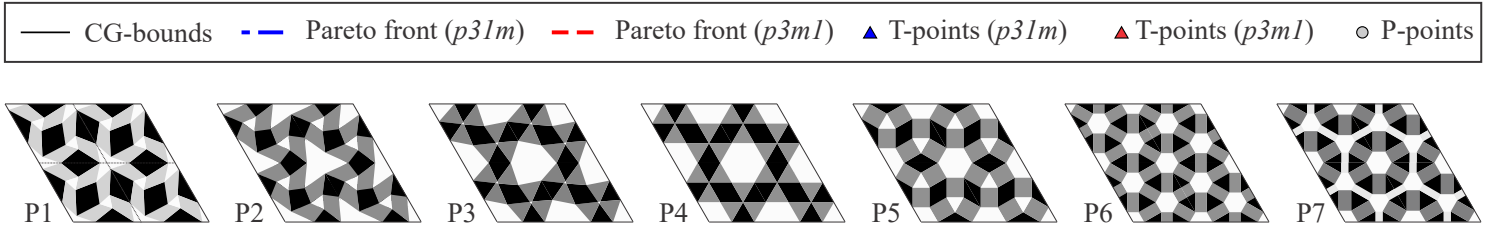

(c)

(d)

\begin{tabular}{|c|c|c|c|c|c|c|c|c|c|}
\hline \multicolumn{3}{|c|}{ Instance } & P1 & P2 & P3 & P4 & P5 & P6 & P7 \\
\hline \multirow{6}{*}{$\begin{array}{l}5 \\
\vdots \\
5 \\
\vdots \\
5 \\
5 \\
5 \\
5 \\
0\end{array}$} & \multirow{2}{*}{$\frac{\Sigma}{\Sigma}$} & $\alpha\left[^{\circ}\right]$ & 36.43 & 48.81 & 97.78 & 120 & - & - & - \\
\hline & & $\gamma[-]$ & 0.20 & 0.52 & 0.54 & 0.50 & - & - & - \\
\hline & \multirow{4}{*}{ §ָ } & $d[-]$ & - & - & - & 0.50 & $5 / 12$ & $1 / 3$ & 0.36 \\
\hline & & $\gamma_{1}[-]$ & - & - & - & 0.50 & 0.50 & 0.50 & 0.56 \\
\hline & & $\gamma_{2}[-]$ & - & - & - & 0.50 & 0.50 & 0.50 & 0.56 \\
\hline & & $\gamma_{3}[-]$ & - & - & - & - & - & 0.50 & 0.10 \\
\hline
\end{tabular}

Figure 7: Near optimal parameterized microstructures. CG-bounds of well-ordered biphasic composites with isotropic properties in the space of effective normalized properties: a) $K$ - $G$, and b) $E$ - $\nu$. Composites with stiff phase volume fraction: $f_{1}=0.5$. Elastic properties of the stiff phase: $E_{1}=1, \nu_{1}=0.3$. Stiffness ratio between phases: $r=2 \times 10^{2}$. c) Family of parameterized microarchitectures with elastic properties close to the CG-bounds. Instances P1 to P7 ( $2 \times 2$ unit cells) d) Parameters defining the geometrical configurations of P1 to P7 microstructures.

Further insight into the obtained microstructures is discussed next. The results displayed by the

P-points, compared with the T-points in Figures $7 \mathrm{a}$ and $\mathrm{b}$, exhibit a notable improvement for 
approaching the extreme properties of the parameterized microstructures. This is the result of including the smaller length scale incorporated by the laminate.

Regarding the configurations with symmetry p31m, effective Poisson's ratios close to the theoretical limits are achieved for the full range of admissible values of $E$ along the Pareto front close to the A-B boundary, such as shown in Figure 7b. The angle $\alpha$ has a strong connection with the effective properties of the composite along this front; the greater the angle $\alpha$, the larger the effective Young modulus, while the parameter $\gamma$ takes an almost constant value, around $\gamma \approx 0.5$. The $\mathrm{P} 4$ configuration, defined by the parameters $\alpha=120^{\circ}$ and $\gamma=0.5$, attains simultaneously the theoretical maximum $K$ and maximum $G$. This configuration constitutes an alternative solution to the Francfort and Murat's rank-3 laminate, although due to the geometrical constraint of the parameterized configuration explained in Section 4.2, it is only correctly defined for $f_{1} \leq 0.5$.

Although the mechanism inducing the auxetic response of the herein reported $p 31 \mathrm{~m}$ architectures resembles the already known rotating triangles structures, or Kagome lattices, the use of laminated areas with variable volume fractions, instead of rigid triangles or bars connected by hinges, defines an alternative microarchitecture.

Solutions generated with the $p 3 m 1$ parametric configurations display a closer approach to the theoretical bounds in the regions denoted A-D and C-D. Configurations obtained with $\gamma_{1}=\gamma_{2}=\gamma_{3}=0.5$ attain the maximum $K$-bound (these composites belong to the class reported by Sigmund [15]). A further property of the present parameterization is that a change of the parameter $d$ induces a modification of the effective shear modulus $G$, allowing to move along the right CG-bound in the $K-G$ space. This effect is observed in the $\mathrm{P} 5$ and $\mathrm{P} 6$ configurations of Figure 7 .

Microstructures P1 and P7 reveal that in the transitions from points B to C, and D to C, respectively, a decrement of the laminate volume fraction is required. Thus, optimal parameterized configurations attaining the lower-left corner of the $K-G$ space tend to topologies with disconnected stiff areas.

The complete set of parameterized microstructures with properties lying on the Pareto fronts in Figures $7 \mathrm{a}$ and $\mathrm{b}$ is presented in a supplementary .csv file and in a supplementary material video (see Data availability Section).

Analysis of the results with stiffness ratio between phases going to infinite. The solutions of additional multi-objective optimization problems with increasing stiffness ratios, $r$, between both phases of the composites, reveal that the optimal parameterized microstructures have effective elastic parameters that are much closer to the CG-bounds if compared with the solution having a lower stiffness ratio $r$. Pareto fronts obtained for composites with $r=2 \times 10^{4}$ and $f_{1}=0.5$ are shown 
in Figure 8a and b with blue and red dashed lines. From these results, it can be seen that the CG-bounds are almost reached throughout their entire extension, except in the top-left corner in the $K-G$ space. Also, a variety of structures with the minimum Poisson's ratio $\nu=-1$ and a wide range of $G$ values can almost be attained.

Next, attention is paid to the case of $p 31 \mathrm{~m}$ parameterization to obtain the left and upper regions of the CG-bounds. Let examine the variation of the optimum parameter $\alpha$ with increasing stiffness ratio $r$ while the parameter $\gamma$ is hold fixed. These results are illustrated in Figure 9, where three set of parameters $\alpha=50^{\circ}, 110^{\circ}$ and $120^{\circ}$ are assessed, and the stiffness ratio is increased from $r=200,500$ to $2 \times 10^{10}$. In the figure, instances a, b and $\mathrm{c}$ for every set of $\alpha$-parameter is indicated with an identical color to that identifying the respective $r$-parameter. The solution with $\alpha=120^{\circ}$ reaches the theoretically stiffest composite, independently of $r$. The solution with $\alpha=50^{\circ}$ achieves auxetic composites. However, for the specific cases of solutions with $\alpha=110^{\circ}$, there is observed a transition from solutions with high $K$ and high $G$ moduli, for low $r$, to solutions with low $K$ and high $G$ moduli, for high $r$.

\section{Microstructures with a single length scale}

Based on the previous discussion and with the objective to attain manufacturable microarchitectures, it is performed further numerical research related to the solutions obtained with the $p 31 \mathrm{~m}$ geometrical configurations.

The laminate material is replaced by a small number of bars with finite thickness. The thickness of the bars are defined to maintain the volume fraction $\gamma$ of the removed laminate unchanged. The transition from the laminate material to a 3-bars configuration is depicted in Figures $8 \mathrm{c}$ and $\mathrm{d}$, respectively. With these bar configurations, new multi-objective optimizations are performed to obtain the corresponding Pareto fronts in the regions $\mathrm{A}-\mathrm{B}$ and $\mathrm{B}-\mathrm{C}$ of the $\mathrm{CG}-\mathrm{bounds}$. One Pareto front is obtained for each number of adopted bars. The results are depicted in Figures $8 \mathrm{a}$ and $\mathrm{b}$ for configurations with 3,9 , and 27 bars.

The solutions move away from the CG-bounds when the bar number decreases, indicating that the bar bending stiffness plays a central role to achieve auxetic behavior and high $G$ values. It was observed that, along the $\mathrm{A}-\mathrm{C}$ region of the $\mathrm{CG}$-bounds, the increase in the number of bars, while holding the parameters fixed, decreases the effective bulk modulus, while the shear modulus is not substantially modified. Thus, the higher the bar number, the lower the bending bar stiffness, the lower the bulk modulus, and the closer to the extreme properties. 


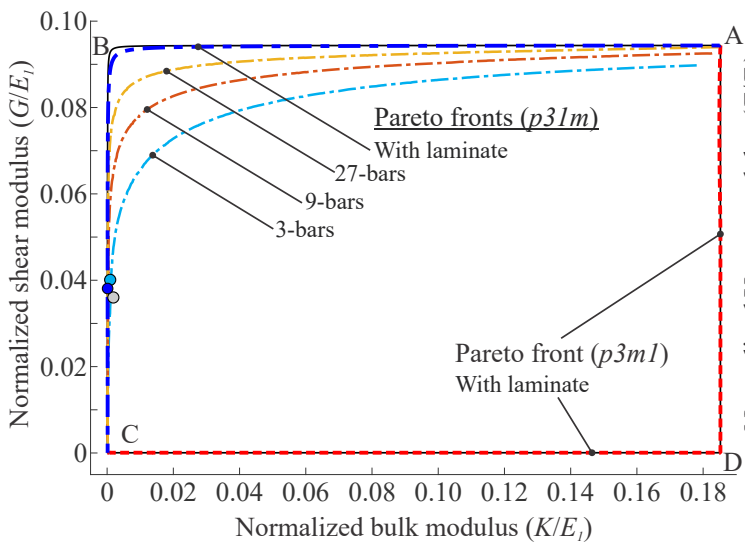

(a)

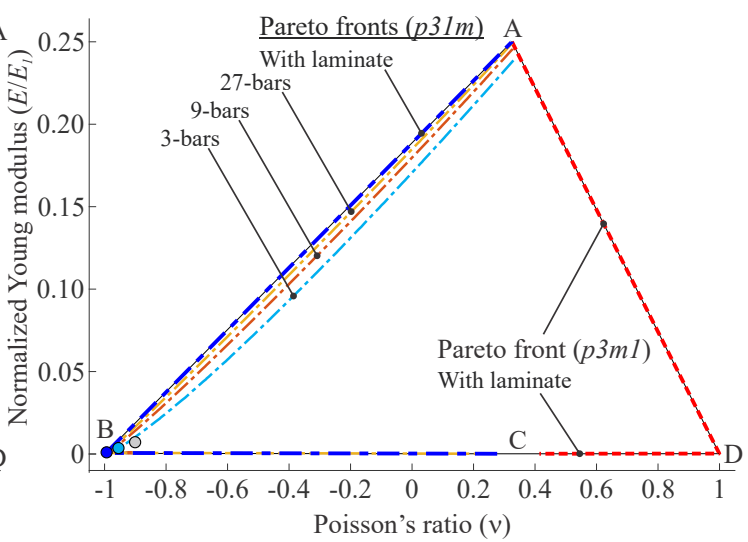

(b)
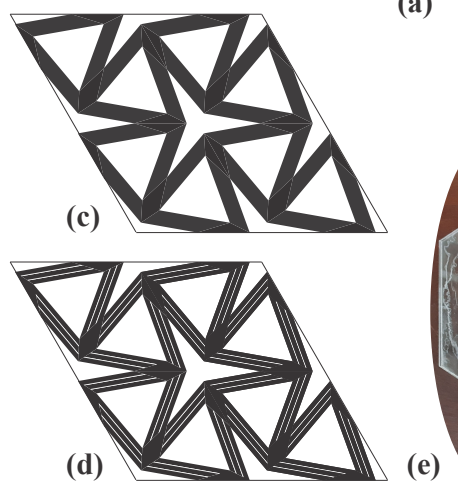

\begin{tabular}{|lr|}
\hline CG-bounds $\bullet$ Numerical homogenization (with laminate) \\
- Numerical homogenization (3-bars) & o Experimental test
\end{tabular}

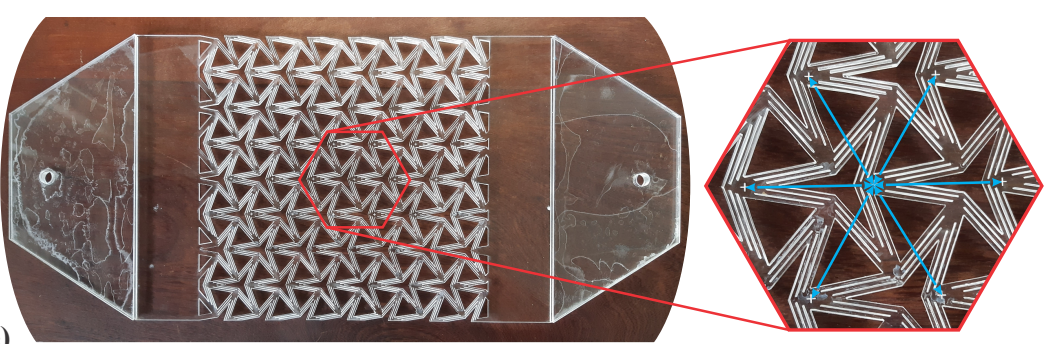

Figure 8: Composites with stiff phase volume fraction: $f_{1}=0.5$. Elastic properties of the stiff phase: $E_{1}=1, \nu_{1}=0.3$. Stiffness ratio between phases: $r=2 \times 10^{4}$. Spaces of normalized a) $K-G$ and b) $E-\nu$. Configurations with $p 31 \mathrm{~m}$ symmetry, $\alpha=20^{\circ}$ and $\gamma=0.9$ ( $2 \times 2$ unit cells): c) with laminate material and d) with three bars; e) Manufactured auxetic composite. Details of the points taken for measuring the relative displacement vectors.

Experimental test of an auxetic microstructure. A specimen is manufactured with the microstructure of Figure 8d, with parameters $\alpha=20^{\circ}$ and $\gamma=0.9$, and is subjected to a tensile test to validate the previous numerical results.

The effective properties for this optimal microstructure, adopting a laminate component (Figure 8c), result as shear modulus $G=0.03799$ and Poisson's ratio $\nu=-0.99998$. If the laminate material is replaced by three bars (Figure 8d), the effective properties obtained are shear modulus $G=0.04009$ and Poisson's ratio $\nu=-0.95481$. These two points are shown in Figure 8a and b as blue and light blue dots respectively. Thus, the relative errors obtained between the 3-bars approximation of the laminate and the optimal microstructure that correspond to a laminate phase are $\epsilon_{G}=5,53 \%$ and $\epsilon_{\nu}=4,52 \%$ for the shear modulus and the Poisson's ratio respectively.

A PMMA sheet of $3 \mathrm{~mm}$ thickness has been cut with a laser beam, furnishing the specimen depicted in Figure 8e. The effective area of the metamaterial is $228 \mathrm{~mm} \times 197 \mathrm{~mm}$, counting with 48 unit 


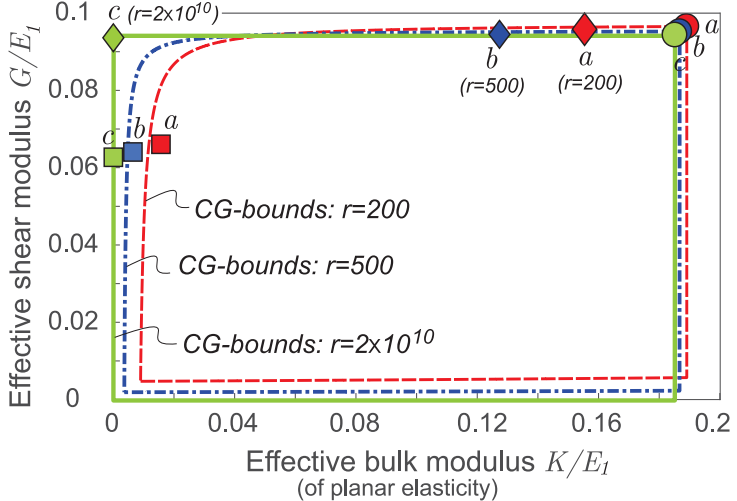

(a)

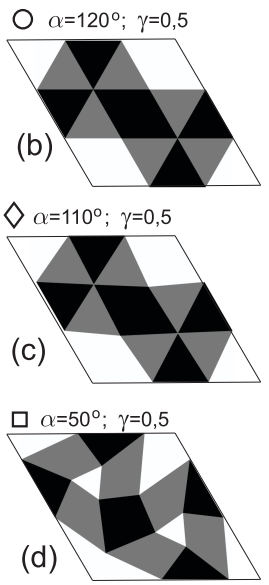

(d)

Figure 9: Analysis of the influence of stiffness ratio between phases on the effective properties. Composites with stiff phase volume fraction $f_{1}=0.5$, and laminate volume fraction $\gamma=0.5$. Elastic properties of the stiff phase: $E_{1}=1$, $\nu_{1}=0.3$. a) CG-bounds in the space of normalized $K-G$ for three stiffness ratios: $r=200$ in red, $r=500$ in blue and $r=2 \times 10^{10}$ in green and effective properties for every stiffness ratio between phases of the displayed microstructures. Microarchitectures defined for: b) $\alpha=120^{\circ}$, c) $\alpha=110^{\circ}$, d) $\alpha=50^{\circ}$.

cells. The Young modulus of the PMMA sheet, $E_{\mathrm{PMMA}}=1.8 \mathrm{GPa}$, is used to normalize the effective Young modulus of the manufactured composite.

Through numerical assessment, it is checked that the loaded specimen displays a reasonably periodic displacement field in its central part. This condition guarantees a reliable measurement of the effective properties in that region. Also, the numerical solution shows that the effective strains on the plane of the sheet are much larger than the transverse strains guaranteeing the plane strain condition assumed in this work.

The relative displacement vectors between equivalent points of the periodic microstructure, in the central region of the specimen, shown in the detail of Figure 8e, are monitored with a Digital Image Correlation (DIC) technique. These displacements furnish an effective strain tensor which is finally used to assess the effective Young modulus and Poisson's ratio. The effective properties assessed in the specimen with this technique are plotted in Figures 8a and b as a gray dot. The Poisson's ratio is -0.9007 and the normalized shear modulus is 0.0360 . Then the relative errors obtained between the experimental results and the Pareto front result that correspond to a laminate phase are $\epsilon_{G}=5,53 \%$ and $\epsilon_{\nu}=9,93 \%$ for the shear modulus and the Poisson's ratio respectively. 


\section{Conclusions}

A microarchitecture design methodology, based on crystal symmetries particularly addressed to attain extreme materials involving three sequential procedures, is presented. It involves a topology optimization stage, formulated as an inverse homogenization problem, the symmetry sensitivity analysis, and the pattern identification stage together with a parameterization of the microarchitecture geometrical configuration. Both the topology optimization and the symmetry sensitivity analysis to capture the CG-bounds have been adressed in previous works by the authors and their main aspects and conclusions has been summarized in this paper. The incorporation of crystal symmetries as an additional constraint allows obtaining simpler configurations with isotropic properties at the first design stage. This guiding rule also makes much easier the posterior topology pattern identification. Remarkably, for the analyzed target, only two crystallographic plane group symmetries are enough to approximate the entire CG-bounds. Geometrical parameterizations characterized by two length scales are proposed. This feature has a significant influence to attain extreme properties. The extreme effective material properties attainable by the parameterized microstructures (Pareto-fronts) are evaluated through multi-objective optimization providing a broad spectrum of possible microstructures with the closest properties to the CG-bounds. For composites of stiff and void phases, the optimum structures expand over almost the full theoretical bounds. As a notable structure, a new solution with simultaneous maximum bulk and shear moduli and only two well-differentiated length scales, is reported for volume fractions, at most, of 0.5, regardless of the contrast between constituent phases. Manufacturability is included in the study, in the sense that simpler geometrical configurations characterized by a single length scale are evaluated. An experimental test is performed on an auxetic composite with a high shear modulus. The test agrees with the numerical predictions. Finally, the potential of this methodology lies in the three prescribed sequential actions providing a practical guide for designing more challenging problems, such as 3D microarchitectures. In these cases, it is rather complex to achieve simple parameterized geometries without the support provided by topology optimization jointly with the imposition of symmetry constraints.

\section{Acknowledgements}

The authors are grateful to Juan Marcos Banegas and Javier Andrés Acosta of CIESE-UTN FRSF for their help with the experimental study. 


$$
\begin{array}{r}
\Omega_{\text {lam }}=6 \times\left(\frac{\sqrt{3} / 2}{\sin \left(120^{\circ}-\alpha / 2\right)+\sin (\alpha / 2)} \times L \times t-\frac{\sin \left(\beta_{1}+60^{\circ}-\alpha / 2\right)}{\sin \left(\beta_{1}+90^{\circ}-\alpha / 2\right)} \times t^{2}-\frac{\sin \left(\beta_{2}-60^{\circ}+\alpha / 2\right)}{\sin \left(\beta_{2}-30^{\circ}+\alpha / 2\right)} \times t^{2}\right), \\
\Omega_{1}=\frac{3}{2} \sin \left(30^{\circ}+\alpha / 2\right) \times\left(\frac{1}{\sin (\alpha / 2)}+\frac{1}{\cos \left(30^{\circ}-\alpha / 2\right)}\right) \times t^{2}, \\
\Omega_{\mu}=\frac{\sqrt{3}}{2} L^{2} .
\end{array}
$$

434

435

\section{APPENDIX: Equations used in the multi-objective optimization problems}

In the mathematical formulation of the multi-objective problem (10), the volume fraction constraints are written as follows.

In the case of $p 31 m$ microstructure, replacing (11) into (10):

$$
\gamma \Omega_{\text {lam }}(\alpha, t)+\Omega_{1}(\alpha, t)-f_{1}^{t} \Omega_{\mu}=0,
$$

where,

The angles $\beta_{1}$ and $\beta_{2}$ depend on $\alpha$, and are calculated from the following expressions:

$$
\begin{array}{r}
\tan \left(\beta_{1}\right)=\frac{3-2 \times \sin \left(30^{\circ}+\alpha\right)}{\sqrt{3}-2 \times \cos \left(30^{\circ}+\alpha\right)}, \\
\tan \left(\beta_{2}\right)=\frac{3+2 \times \cos (\alpha)}{\sqrt{3}+2 \times \sin (\alpha)} .
\end{array}
$$

In the case of the $p 3 m 1$, by replacing (12) into (10):

$$
\gamma_{1} \Omega_{l a m 1}(d, t)+\gamma_{2} \Omega_{l a m 2}(d, t)+\gamma_{3} \Omega_{l a m 3}(d, t)+\Omega_{1}(d, t)-f_{1}^{t} \Omega_{\mu}=0,
$$

${ }^{436}$ where $\gamma_{1}, \gamma_{2}, \gamma_{3}$ and $f_{1}^{t}$ are parameters defined in Figure 5 and

$$
\begin{array}{r}
\Omega_{\text {lam } 1}=\Omega_{\text {lam } 2}=3 \times d \times L \times t-\sqrt{3} \times t^{2}, \\
\Omega_{\text {lam } 3}=3 \times(L-2 \times d \times L) \times t-\sqrt{3} \times t^{2}, \\
\Omega_{1}=\frac{3 \sqrt{3}}{2} t^{2}, \\
\Omega_{\mu}=\frac{\sqrt{3}}{2} L^{2} .
\end{array}
$$

The $d$ parameter may increase beyond the vanishing of the $\gamma_{3}$ laminate region (see for example microstructures P4 and P5 in Figure 7). in this situation the relation between the parameters to respect a given volume fraction comes from:

$$
\gamma_{1} \Omega_{\text {lam } 1}(d, t)+\gamma_{2} \Omega_{l a m 2}(d, t)+\Omega_{1}(d, t)-f_{1}^{t} \Omega_{\mu}=0,
$$

where

$$
\begin{array}{r}
\Omega_{l a m 1}=\Omega_{l a m 2}=3 \times(L-d \times L) \times t-2 \times \sqrt{3} \times t^{2}, \\
\Omega_{1}=2 \times \sqrt{3} \times t^{2}-6 \times \sqrt{3} \times(L / 2-d \times L)^{2} .
\end{array}
$$




\section{Declarations}

Funding: CONICET and ANPCyT - Argentina (grants PICT 2016-2673).

Conflict of Interest: The authors declare that they have no conflict of interest.

Availability of data and material: A video showing the transition of the microstructures attained along the four Pareto fronts, and a .csv file describing the values of the parameters and properties of all the microstructures lying on the Pareto fronts, can be found in the open-source online data repository hosted at Mendeley Data [46].

Code availability: the homemade code is written in Matlab and is freely available under request.

\section{Replication of results}

The homemade codes, including the inverse homogenization of Section 3, the geometrical description of the parameterized microstructures with conformal meshes of Section 4 and the multiobjective optimization using a genetic algorithm of Section 5, have been implemented in Matlab. Numerical data characterizing the presented results, for instance, material properties of the phases, and optimal parameters are displayed in the paper. The full set of numerical and experimental data, as well as the codes are freely available under request.

[1] J. Liu, A. T. Gaynor, S. Chen, Z. Kang, K. Suresh, A. Takezawa, L. Li, J. Kato, J. Tang, C. Wang, et al., Current and future trends in topology optimization for additive manufacturing, Structural and Multidisciplinary Optimization 57 (6) (2018) 2457-2483.

[2] C. Imediegwu, R. Murphy, R. Hewson, M. Santer, Multiscale structural optimization towards three-dimensional printable structures, Structural and Multidisciplinary Optimization 60 (2) (2019) 513-525.

[3] A. Cherkaev, L. Gibiansky, Coupled estimates for the bulk and shear moduli of a twodimensional isotropic elastic composite, J. Mech. Phys. Solids 41 (5) (1993) 937-980. doi: $10.1016 / 0022-5096(93) 90006-2$.

[4] Z. Hashin, On elastic behaviour of fibre reinforced materials of arbitrary transverse phase geometry, Journal of the Mechanics and Physics of Solids 13 (3) (1965) 119-134.

[5] G. Milton, A. Cherkaev, Which elasticity tensors are realizable?, J. Eng. Mater. Technol. 117 (4) (1995) 483-493. 
[6] A. Norris, Acoustic cloaking theory, in: Proceedings of the Royal Society of London A: Mathematical, Physical and Engineering Sciences, Vol. 464, 2008, pp. 2411-2434.

[7] Y. Cheng, F. Yang, J. Xu, X. Liu, A multilayer structured acoustic cloak with homogeneous isotropic materials, Appl. Phys. Lett. 92 (15) (2008) 151913.

[8] C. Layman, C. Naify, T. Martin, D. Calvo, G. Orris, Highly anisotropic elements for acoustic pentamode applications, Phys. Rev. Lett. 111 (2) (2013) 024302.

[9] C. Méndez, J. Podestá, O. Lloberas-Valls, S. Toro, A. Huespe, J. Oliver, Computational material design for acoustic cloaking, Int. J. Numer. Methods Eng. 112 (10) (2017) 13531380.

[10] G. Milton, Planar polycrystals with extremal bulk and shear moduli, arXiv preprint arXiv:2002.08788.

[11] Y. Wang, O. Sigmund, Quasiperiodic mechanical metamaterials with extreme isotropic stiffness, Extreme Mechanics Letters 34 (2020) 100596. doi:10.1016/j.eml.2019.100596.

[12] Z. Hashin, The elastic moduli of heterogeneous materials, J. Appl. Mech. 29 (1) (1962) 143150. doi:10.1115/1.3636446.

[13] Z. Hashin, S. Shtrikman, A variational approach to the theory of the elastic behaviour of multiphase materials, J. Mech. Phys. Solids 11 (2) (1963) 127-140. doi:10.1016/0022-5096(63) 90060-7.

[14] S. Vigdergauz, Energy-minimizing inclusions in a planar elastic structure with macroisotropy, Struct. Optim. 17 (2-3) (1999) 104-112. doi:10.1007/BF01195935.

[15] O. Sigmund, A new class of extremal composites, J. Mech. Phys. Solids 48 (2) (2000) 397-428. doi : 10.1016/S0022-5096(99)00034-4.

[16] A. Norris, A differential scheme for the effective moduli of composites, Mech. Mater. 4 (1) (1985) 1-16. doi:10.1016/0167-6636(85)90002-X.

[17] G. Milton, Modelling the properties of composites by laminates, in: Homogenization and effective moduli of materials and media, Springer, 1986, pp. 150-174. doi:10.1007/ 978-1-4613-8646-9_7.

[18] G. A. Francfort, F. Murat, Homogenization and optimal bounds in linear elasticity, Arch. Ration. Mech. Anal. 94 (4) (1986) 307-334. doi:10.1007/bf00280908. 
[19] G. Milton, M. Briane, D. Harutyunyan, On the possible effective elasticity tensors of 2dimensional and 3-dimensional printed materials, Math. Mech. Complex Syst. 5 (1) (2017) 41-94. doi:10.2140/memocs . 2017.5.41.

[20] G. W. Milton, Some open problems in the theory of composites, arXiv preprint arXiv:2008.03394.

[21] S. Babaee, J. Shim, J. C. Weaver, E. R. Chen, N. Patel, K. Bertoldi, 3d soft metamaterials with negative poisson's ratio, Adv. Mater. 25 (36) (2013) 5044-5049. doi:10.1002/adma. 201301986.

[22] T. Bückmann, R. Schittny, M. Thiel, M. Kadic, G. W. Milton, M. Wegener, On threedimensional dilational elastic metamaterials, New J. Phys. 16 (3) (2014) 033032. doi: $10.1088 / 1367-2630 / 16 / 3 / 033032$.

[23] M. Rupin, P. Roux, G. Lerosey, F. Lemoult, Symmetry issues in the hybridization of multimode waves with resonators: an example with lamb waves metamaterial, Sci. Rep. 5 (2015) 13714. doi:10.1038/srep13714.

[24] Z. Wu, Y. Zheng, Moiré chiral metamaterials, Adv. Opt. Mater. 5 (16) (2017) 1700034. doi: 10.1002/adom. 201700034.

[25] T. Frenzel, M. Kadic, M. Wegener, Three-dimensional mechanical metamaterials with a twist, Science 358 (6366) (2017) 1072-1074. doi:10.1126/science.aao4640.

[26] H. Zong, H. Zhang, Y. Wang, M. Y. Wang, J. Y. Fuh, On two-step design of microstructure with desired poisson's ratio for am, Materials \& Design 159 (2018) 90-102.

[27] X. Ni, X. Guo, J. Li, Y. Huang, Y. Zhang, J. A. Rogers, 2d mechanical metamaterials with widely tunable unusual modes of thermal expansion, Adv. Mater. 31 (48) (2019) 1905405. doi:10.1002/adma. 201905405.

[28] T. Frenzel, J. Köpfler, E. Jung, M. Kadic, M. Wegener, Ultrasound experiments on acoustical activity in chiral mechanical metamaterials, Nat. Commun. 10 (1) (2019) 1-6. doi:10.1038/ s41467-019-11366-8.

[29] M. Kadic, G. W. Milton, M. van Hecke, M. Wegener, 3d metamaterials, Nat. Rev. Phys. 1 (3) (2019) 198-210. doi:10.1038/s42254-018-0018-y. 
[30] O. Sigmund, Materials with prescribed constitutive parameters: an inverse homogenization problem, Int. J. Solids Struct. 31 (17) (1994) 2313-2329. doi:10.1016/0020-7683(94) 90154-6.

[31] J. Podestá, C. Méndez, S. Toro, A. Huespe, Symmetry considerations for topology design in the elastic inverse homogenization problem, J. Mech. Phys. Solids 128 (2019) 54-78. doi: $10.1016 / j \cdot j m p s .2019 .03 .018$.

[32] C. Méndez, J. Podestá, S. Toro, A. Huespe, J. Oliver, Making use of symmetries in the three-dimensional elastic inverse homogenization problem, Int. J. Multiscale Comput. Eng. 17 (2019) 261-280. doi:10.1615/Int JMultCompEng. 2019029111.

[33] R. Yera, N. Rossi, C. Méndez, A. Huespe, Topology design of 2d and 3d elastic material microarchitectures with crystal symmetries displaying isotropic properties close to their theoretical limits, Appl. Mater. Today 18 (2020) 100456. doi:10.1016/j.apmt.2019.100456.

[34] N. Rossi, R. Yera, C. Méndez, S. Toro, A. Huespe, Numerical technique for the 3d microarchitecture design of elastic composites inspired by crystal symmetries, Comput. Methods Appl. Mech. Eng. 359 (2020) 112760. doi:10.1016/j.cma.2019.112760.

[35] O. Sigmund, K. Maute, Topology optimization approaches, Struct. Multidiscip. Optim. 48 (6) (2013) 1031-1055.

[36] L. Xia, P. Breitkopf, Design of materials using topology optimization and energy-based homogenization approach in matlab, Structural and Multidisciplinary Optimization 52 (6) (2015) $1229-1241$.

[37] M. Osanov, J. Guest, Topology optimization for architected materials design, Annu. Rev. Mater. Sci. 46 (2016) 211-233.

[38] S. Amstutz, H. Andrä, A new algorithm for topology optimization using a level-set method, J. Comput. Phys. 216 (2) (2006) 573-588.

[39] S. Amstutz, S. Giusti, A. Novotny, E. de Souza Neto, Topological derivative for multi-scale linear elasticity models applied to the synthesis of microstructures, Int. J. Numer. Methods Eng. 84 (6) (2010) 733-756.

[40] S. Amstutz, Connections between topological sensitivity analysis and material interpolation schemes in topology optimization, Structural and Multidisciplinary Optimization 43 (6) (2011) $755-765$. 
[41] A. Novotny, J. Sokołowski, Topological derivatives in shape optimization, Interaction of Mechanics and Mathematics, Springer Science \& Business Media, 2013.

[42] S. M. Giusti, Z. Mróz, A. Novotny, J. Sokołowski, Topology design of thermomechanical actuators, Structural and Multidisciplinary Optimization 55 (5) (2017) 1575-1587.

[43] P. J. Blanco, P. J. Sánchez, E. A. de Souza Neto, R. A. Feijóo, Variational foundations and generalized unified theory of rve-based multiscale models, Archives of Computational Methods in Engineering 23 (2) (2016) 191-253.

[44] I. Ostanin, G. Ovchinnikov, D. Tozoni, D. Zorin, A parametric class of composites with a large achievable range of effective elastic properties, J. Mech. Phys. Solids 118 (2018) 204217. doi:10.1016/j.jmps.2018.05.018.

[45] K. Deb, A. Pratap, S. Agarwal, T. Meyarivan, A fast and elitist multiobjective genetic algorithm: Nsga-ii, IEEE Trans. Evol. Comput. 6 (2) (2002) 182-197. doi:10.1109/4235.996017.

[46] N. Rossi, J. Podestá, F. Bre, C. Méndez, A. Huespe, Dataset of parameterized isotropic microstructures attaining extreme properties, Mendeley Data, v1 http://dx.doi.org/10.17632/hcpvmnwm4p.1. 